\title{
CIENCIA, TECNOLOGÍA Y EDUCACIÓN EN COSTA RICA EN EL PERÍODO 2010-2014
}

\section{SCIENCE, TECHNOLOGY AND EDUCATION IN COSTA RICA BETWEEN 2010 AND 2014}

\author{
Rafael Evelio Granados Carvajal \\ Oscar David Calvo Solano ${ }^{2,3}$
}

Fecha de recepción: 2 de marzo de 2017 - Fecha de aceptación: 26 de junio de 2017

\begin{abstract}
Resumen
En este artículo se analizaron las distintas redes vinculadas a la formulación de políticas públicas en la Comisión de Ciencia y Tecnología del Congreso de Costa Rica, entre el 2010 y 2014, con el propósito de describir el abordaje realizado y la incidencia sobre el estilo de desarrollo costarricense. Se encontró que las principales redes presentan propuestas cerradas y un Poder Ejecutivo con más cercanía a los distintos sectores, pero no suficiente. A raíz de lo anterior, se concluye que existe una limitada coordinación y cohesión de los actores relacionados con la ciencia, la tecnología y la educación en Costa Rica, determinando limitados alcances en educación, empleo, competitividad y desarrollo; especialmente, pensando en la dinámica que ejercen la ciencia y la tecnología en el mediano y largo plazo en el proceso de desarrollo económico y social.

Palabras clave: Rumbo, Políticas públicas, Redes sociales, Coordinación, Cohesión.
\end{abstract}

\begin{abstract}
On planning the economic course of a country, it is necessary to invest in science, technology and education, due to contribute to produce human resources oriented to an optimal development. So, the establishment of public policies by the government with the contribution of related actors creating a social network between both is important to optimize such process. Networks related to public policies formulation in the Science and Technology Committee in the Costa Rican Congress and its relationship with related actors were analyzed due to verify if their approach have a significant impact in Costa Rican society. Closeness between the government and different groups, and a short point of view in the proposals given by related actors were found. We concluded, that there is a weak relationship and a limited coordination between the main actors related with Science, Technology and Education in Costa Rica.
\end{abstract}

Key Words: Course, Public policies, Social networks, Coordination, Cohesion.

1 Programa Regional de Desarrollo Rural (PRDR). Universidad Nacional de Costa Rica (UNA), rafagranado@gmail.com

2 Programa de Estudios Sociales de la Ciencia, la Técnica y el Medio Ambiente (PESCTMA). Centro de Investigaciones Geofísicas (CIGEFI). Universidad de Costa Rica (UCR), oscar.calvosolano@ucr.ac.cr

3 Instituto de Física Teórica (IFT). 


\section{Introducción}

Esta investigación analiza la dinámica seguida sobre Ciencia, Tecnología y Educación (CTE) del Congreso costarricense, como parte de un proceso de debate con respecto a las políticas públicas en este campo. Se presentan las diferentes redes y perspectivas de este tema que generan controversias, con el objetivo de orientar la elaboración de las políticas públicas e implementarlas.

A lo largo de la historia, la ciencia y la tecnología han generado una gran expectativa debido a las aplicaciones que pueden mejorar la calidad de vida; pero también, han provocado muchas controversias cuando son utilizadas con fines destructivos o cuando existen recursos limitados que pueden ser causa de mayores problemas. Por lo tanto, la gestión de las políticas ligadas a la ciencia y la tecnología encierra un espacio importante en la estructura funcional del Estado moderno y genera problemas importantes para actores políticos implicados.

Desde 1990, Costa Rica cuenta con una Ley de Ciencia y Tecnología que provoca una ruptura formal con un pasado, donde la ciencia y la tecnología dependían más de procesos parciales entre las instituciones y el Gobierno. En este nuevo período, se da una transición en la dinámica del patrón tecnológico y la gestión del Estado, que impactan tanto una tendencia a un sistema nacional de innovación, como a un nuevo dinamismo de la ciencia y tecnología, que es parte del proceso de globalización.

Para orientar la investigación, se plantean varias preguntas desde la perspectiva de los estudios sociales de la ciencia, la tecnología y la innovación: ¿En la segunda década del siglo XXI cuántas redes que desarrollan controversia sobre la temática de ciencia y tecnología, llegan a la arena política del congreso? ¿Cómo se reorganizan y agrupan los diferentes actores estratégicos de la ciencia y la tecnología? ¿Cuáles son los principales proyectos que se discuten en el tema? ¿Cuáles son los sectores sociales y productivos incorporados en la discusión? ¿Es posible, como país, escoger una ruta de crecimiento económico articulando las distintas visiones para superar las desigualdades sociales, garantizando mayores niveles de empleo, competitividad y desarrollo social?

\section{Aspectos teóricos}

Para el análisis de políticas públicas de ciencia y tecnología se pueden encontrar diversas propuestas tradicionales, las más clásicas son aquellas que se enfocan en hacer una división entre lo público y la privado (Gomá \& Subirats, 1998) y la segunda común, es aquella que centra su análisis en los diferentes niveles de la estructura de gobierno. No obstante, como reconocen Gomá y Subirats (1998) la división entre estas dos posibilidades está cada vez más erosionada, pero se está avanzando en un análisis que da cuenta de la interacción entre actores e instituciones, donde surgen negociaciones en la arena política; ahí se entreteje la negociación y se da la controversia entre las diferentes redes de actores que actúan en ambas esferas. Lo anterior propone que el análisis de estas políticas públicas debe partir de una perspectiva de relaciones entre redes.

La erosión se da ante una perspectiva que reconoce el top down como una forma de gobernar que ha llegado a sus límites. En los inicios del siglo XXI, la democracia demandó la búsqueda de nuevos mecanismos de gobernanza y pluralismo en la formulación y gestión de las políticas públicas en general; sin embargo, las características actuales son otras, se reclama mayor transparencia, eficiencia y eficacia en el Estado, razón por la que la ciencia y tecnología empiezan a jugar un papel diferente y más complejo, se proyectan estas políticas en perspectiva de generación de innovación, empleo, competitividad y desarrollo económico y social; en un marco de cohesión social y reducción de las desigualdades.

En el camino de la construcción de proyectos, agendas y negociaciones, muchas iniciativas pasan a la Asamblea Legislativa (AL) y se conducen hacia una comisión del Congreso, especializada en el tema de ciencia y tecnología. Ésta es el resultado de una estructura de Estado y sus diferentes poderes, donde se negocian decisiones en un marco casi de diálogo, sin poder imponer o llegar a 
acuerdos finales; no se controla el comportamiento de las diferentes redes que intervienen ni de los resultados; no obstante, la Comisión constituye un filtro importante para conocer rumbos y perspectivas de ciencia y tecnología.

"Las comisiones están conformadas por grupos deliberativos, integradas por un determinado número de legisladores, que tienen como función conocer y analizar proyectos de ley, cuyo dictamen, informe o decisión son enviados al Plenario Legislativo para que este se pronuncie al respecto" (Asamblea Legislativa, Proyecto de Ley 18773, 2010). En la Comisión, se abre un espacio de discusión amplio, como parte del proceso legislativo aplicado en el análisis de los proyectos de ley para el control político, el estudio y dictamen de los Presupuestos de la República, la investigación, recomendaciones, encuentros con otras instancias del Estado y diferentes redes de actores estratégicos vinculados, con orígenes diversos tales como las cámaras empresariales, el Ministerio de Educación Pública (MEP) y, otros actores organizados sobre estos temas que buscan tener incidencia.

En el proceso de formulación de una ley de interés general o una política pública se imponen escalas de valores y criterios para el análisis de alternativas. Hay una dimensión mediática, procedimientos, instrumentos, rutas críticas de acción, entre otros (Aguilar, 1996). Para el análisis se partirá del concepto de traducción, aportado por una amplia comunidad de científicos de la Teoría del ActorRed (Latour, 1991; Law, 1998; Latour, 1998; Callon, 1986; Doménech \& Tirado, 2008; Arellano, 2003).

Por lo anterior, en el proceso de formulación de políticas es necesario asumir la existencia de una diversidad de actores que, controversialmente, tratan de imponer sus visiones, y es en ese punto cuando surgen relaciones, se retoman posiciones y se establecen acuerdos y logran establecer cada posición en una situación que los demás deben conocer, para luego abrir espacios de encuentro. El debate lleva a las reformas, a los cambios, modificación de lo formulado, de las acciones y alternativas a seleccionar. En este caso, la traducción significa describir el sentido de los diálogos a lo largo de la trayectoria de un proceso, y así comprender el camino seguido por la política, el orden ocurrido y los resultados.

Entonces, surgen preguntas que complementan las anteriores, por lo que interesa conocer cuáles son los actores inmersos en las políticas y el tipo de iniciativas discutidas, cómo se articulan determinados actores de interés, cómo se conforma la estrategia de ciencia y tecnología en Costa Rica, cuáles son los proyectos que llegan y cuáles son priorizados. Eso dará cuenta de cuáles perspectivas se imponen o lograron convencer. "Desde la perspectiva sociotécnica, la capacidad de determinados actores para controlar a otros sean seres humanos, instituciones o entidades naturales, o para obedecerlos, depende de una compleja red de interacciones" (Callon, 1998, citado por Grau et al., 2010).

\section{Métodos}

Esta investigación es cualitativa. Se revisaron las actas del Congreso y se determinó cuáles son sus actores estratégicos. Además, se realizaron entrevistas abiertas a actores clave, con el fin de triangular la información. Se tuvo como objetivo conocer las subjetividades sobre ciencia y tecnología a partir de documentos y perspectivas de actores relacionados, en un contexto determinado por la necesidad de articular el rumbo del país con la sociedad del conocimiento y la globalización.

Se describe y reconstruye analíticamente el carácter interpretativo (Barrantes, 2001) que tienen los distintos actores en torno al eje de CTE. Esto a partir de las sesiones ordinarias y extraordinarias sostenidas dentro de la Comisión Legislativa, AL, el Ministerio de Ciencia, Tecnología y Telecomunicaciones (MICITT), el Consejo Nacional de Rectores (CONARE) y otros actores que no participan, pero se encuentran citados y han enviado o analizado propuestas a partir de la solicitud de la Comisión.

Se estudiaron los proyectos más importantes sobre CTE. Además, se indagó sobre cómo se formularon para crear condiciones de gestión de las políticas públicas y cuáles son los actores y las entidades involucradas que tienen un espacio en la arena política del Congreso, para incidir o disentir sobre las tendencias impulsadas. 
Se realizó también un análisis de redes, conectando las perspectivas en materia de CTE entre cada uno de los actores. Esto, con el propósito de encontrar diferencias y similitudes que influencien el establecimiento de las políticas en torno a diferentes ejes: educativos, inversión, culturales e innovación.

\section{Discusión}

\section{Antecedentes contextuales a las políticas de CTE}

Desde finales de la década del sesenta, Costa Rica mejoró sus tendencias de desempeño económico con una estrategia que buscaba articularse al mercado mundial, mediante la producción de bienes de origen agrícola. Más tarde, incursiona en forma limitada hacia el desarrollo industrial y, en los años noventa, profundizó su especialización en la venta de servicios y planteó acciones para atraer inversión extranjera, producir bienes tecnológicos e incentivar el turismo y la protección del medio ambiente. Esto permitió llevar a cabo una diversificación de la economía que, le permitió alcanzar estabilidad política y mantener un desempeño económico estable. No obstante, los índices de pobreza y desigualdad social han empeorado, en un contexto similar al de América Latina, debido a que el Estado no logró mejorar la eficacia en la gestión de políticas públicas sociales.

Los principales desafíos para Costa Rica fueron: a) el crecimiento del índice de pobreza y la concentración del ingreso, b) el creciente déficit fiscal y limitadas estrategias para la coordinación de las redes de políticas públicas y c) la escasa articulación entre el sector público y un gran número de debilidades para fortalecer los gobiernos locales. Los debates concentran su atención en las tareas para avanzar hacia una economía descentralizada, con lo cual se procura articular a las organizaciones comunales y a la sociedad civil para dar viabilidad a la gobernanza.

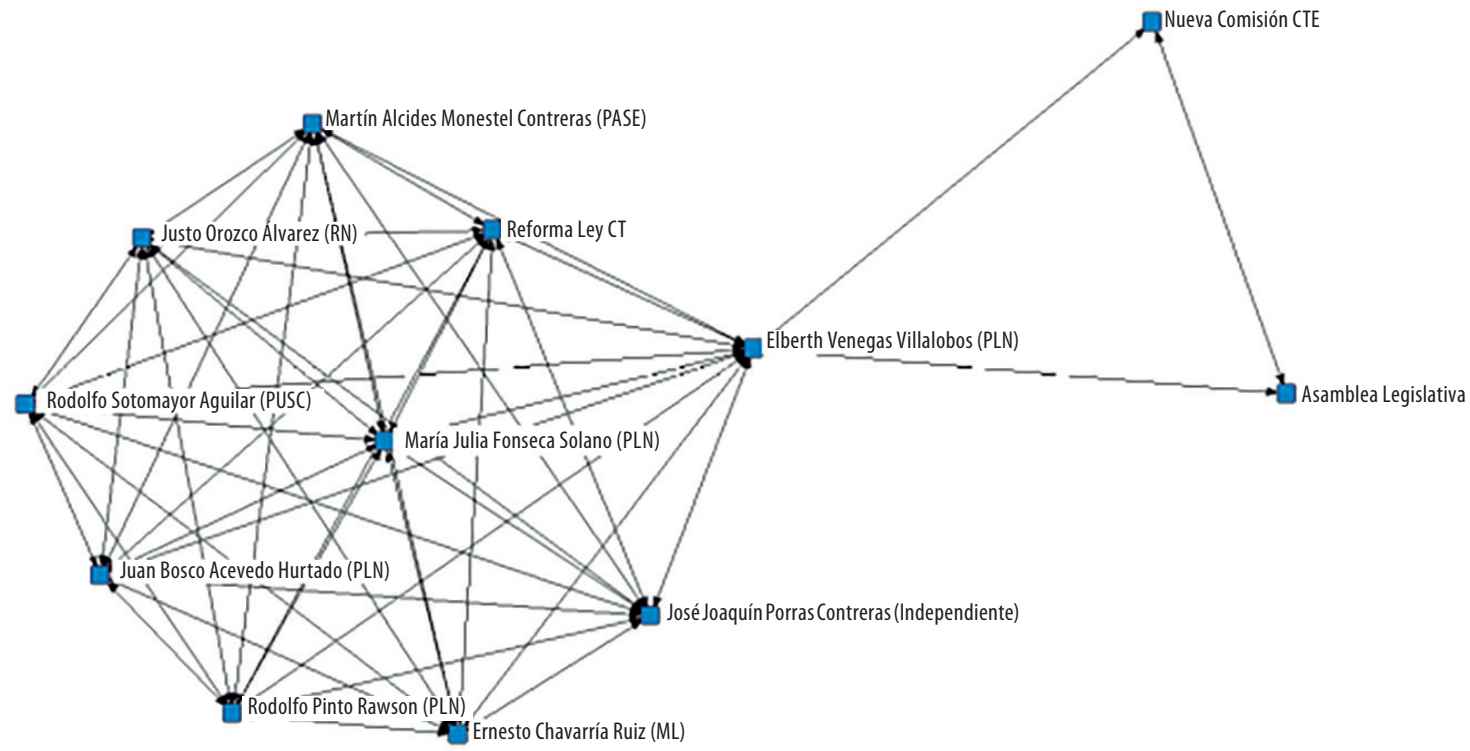


En los diferentes debates académicos y en la esfera de Gobierno se reconoce que los esfuerzos del pasado en materia de CTE son insuficientes, por lo que se requiere de una propuesta integral que vaya desde la escuela hasta la formación del recurso humano en áreas científico-tecnológicas con la perspectiva de "mantener al país competitivo a nivel internacional" (Expediente Legislativo 18773, 2013a). En la AL se forma una Comisión Permanente 4 para discutir las principales propuestas y los diversos apoyos en materia de legislación que se requieren; el orden de la agenda en ciencia y tecnología, en una primera instancia, se deja en manos de la Comisión (Grafo 1).

La Comisión en aquel período estuvo integrada por siete diputados, representantes de cinco fracciones políticas, como se evidencia en la Resolución N. . 08-10-11 de la Presidencia de la AL, siendo Elibeth Venegas Villalobos la primera presidenta de la misma. La primera sesión de la Comisión se llevó a cabo el 22 de junio de 2010. El primer paso, fue revisar los diagnósticos de distintas redes. No obstante, por el estilo de trabajo y experiencia, se van imponiendo las visiones de los organismos internacionales, propuestas por consultores de corte internacional, entre otros.

La red a cargo de la Comisión logró avances importantes, pues, por una parte, aparece como una comisión específica de ciencia y tecnología que evoluciona a una Comisión Permanente de Ciencia, Tecnología y Educación. Por otra parte, representa una intención para modificar la Ley de Ciencia y Tecnología formulada desde 1990.

Sobre lo anterior, el Foro Económico Mundial menciona que “...los elementos que explican el limitado desempeño de la productividad son variados, aunque en la práctica uno de los más importantes se asocia con la insuficiente inversión en innovación. Costa Rica se ha estancado en la inversión en investigación y desarrollo (I+D), manteniéndose alrededor del 0,5\% del PIB, cuando según su nivel de desarrollo debería estar invirtiendo al menos el doble" (Asamblea Legislativa, 2013a). En este discurso, se percibe una atención a los recursos, y poca atención al estilo de gestión de las políticas públicas.

Por otra parte, el Banco Interamericano de Desarrollo (BID), comentó que hay “... otros factores que inhiben una mayor inversión en innovación que incluye, entre los más importantes: (a) escaso capital humano, (b) falta de financiamiento y (c) limitado acceso a prácticas modernas de gestión de calidad y desarrollo empresarial"5. Se argumenta que todo lo anterior se puede encontrar también en los estudios realizados por el MICITT desde el 2007. Dichos estudios, sostienen que faltan incentivos financieros para que la empresa nacional logre innovar, "donde el $45.2 \%$ de las empresas reportan la falta de acceso al financiamiento como la principal barrera a la innovación” (Asamblea Legislativa, 2013a).

Desde este diagnóstico, el Poder Ejecutivo (PE), la AL y el MICITT proponen diferentes estrategias para impulsar leyes, presupuestos y abrir nuevas oportunidades. Un actor central es el CONARE, el cual tiene su propia agenda, fruto de un arduo debate entre cuatro universidades públicas a saber: La Universidad de Costa Rica (UCR), Universidad Nacional de Costa Rica (UNA), Instituto Tecnológico de Costa Rica (ITCR) y Universidad Estatal a Distancia (UNED).

La agenda nacional de CTE va hacia los siguientes temas: a) crear el Consejo Presidencial de Competitividad e Innovación (CPCI) desde el marco del Plan Nacional de Ciencia, Tecnología e Innovación (PNCTI) 2011-2014; b) crear la Comisión Permanente de Ciencia, Tecnología y Educación en el Congreso; c) reformar la ley de Ciencia y Tecnología del año 1990 y d) incrementar el presupuesto

4 Es necesario dedicar un espacio para reconocer la importancia de la figura de la "Comisión" como órgano de gestión política, con una larga historia entre los mecanismos políticos para dejar de hacer o para impulsar políticas públicas en materia de ciencia y tecnología, dependiendo del interés y de los grupos de actores. El concepto de comisión aparece en la historia costarricense desde el año 1824, ligado a la Constitución Política de la República Federal de Centroamérica. En el artículo 73, del reglamento de la AL, indica: "Admitido deberá pasar a una comisión que lo examinará detenidamente" (Asamblea Legislativa, 2014), esto se refiere al proyecto de ley que conociera el Congreso. Dicha figura inició como un recurso político-administrativo, cuya función fue rendir informes que remitía al Congreso para su debate y respectiva toma de decisiones. 
público a la educación. Los temas ordenados se tratan para ampliar diferentes aspectos sobre el origen e intereses de cada una de estas iniciativas y brindar mayor información de los actores estratégicos del proceso.

Es importante recapitular que la Comisión fue formada por diputados de diferentes partidos políticos que representan diversas visiones, y que contaban con diferentes proyectos y trayectorias alejadas a la ciencia y la tecnología. La discusión de iniciativas referidas a CTE, en procura de fortalecer estrategias en dirección al rumbo económico del país no eran su principal cualidad. Esta parecía ser una agenda más de contención que de desarrollo, lo cual parece un gran error político: dejar las agendas de largo plazo en manos de actores que no son idóneos para promover iniciativas en dichos temas.

\section{Creación del CPCI}

El CPCI se crea en el marco del PNCTI para el período 2011-2014, y estuvo orientado por el MICITT. No obstante, es el sector privado quien diseñó las agendas concentradas en temas de infraestructura, simplificación de trámites y en promoción de la atracción de inversiones. Las prioridades desde el 2011 fueron la búsqueda de mecanismos para el desarrollo de capital humano y la innovación. El marco de análisis fue acotado por el aprovechamiento del contexto dado por el comercio exterior, utilizando los tratados comerciales, acuerdos y otros instrumentos vigentes. Los mayores retos para el Gobierno fueron la apertura del sector para satisfacer la demanda energética en un $95 \%$ con fuentes de energía renovable y generar una cultura de coordinación con otras instituciones.

Para impulsar esta iniciativa se unen esfuerzos del Ministerio de Comercio Exterior (COMEX), Consejo de la Promoción de la Competitividad (CPC), el MICITT y la Promotora de Comercio Exterior (PROCOMER). Se trabaja en propuestas discutidas, en primera instancia, por las universidades para formar factor humano y apoyar el proceso innovador en la empresa privada:

\footnotetext{
El objetivo general del Programa es contribuir al crecimiento de la productividad mediante el apoyo a las actividades de innovación del sector productivo y la formación de capital humano avanzado en áreas estratégicas. Estas se refieren a aquellas establecidas por el PNCTI para el 2011-2014 del Gobierno de Costa Rica, tales como tecnologías digitales, nuevos materiales, biotecnología y energías renovables; claves para el estímulo de la innovación a empresas domésticas y la atracción de actividades de innovación por parte de empresas globales y el fomento al emprendimiento de base tecnológica (MICIT, 2011, p. 7).
}

Grafo 2

Red del CPCI, para la innovación y desarrollo de factor humano 2012-2013

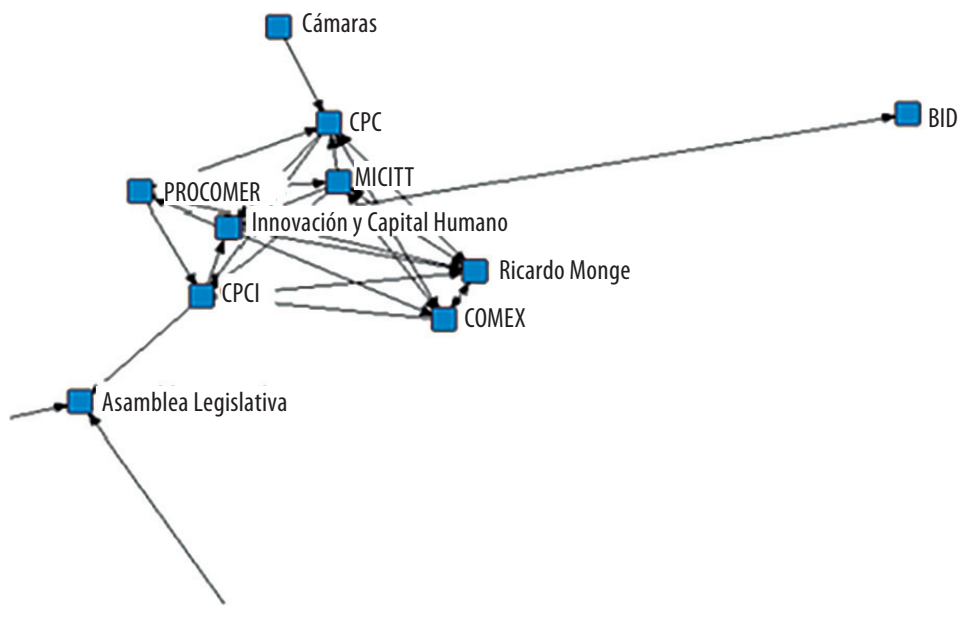


El Consejo partió de un diagnóstico global brindado por los organismos internacionales sin consultar a actores institucionales, regionales y locales. Esto refleja una perspectiva corporativista, una construcción de propuestas de arriba hacia abajo, que limita mayores alcances a los proyectos debido a la exclusión de otras visiones.

\section{Reformar la Ley de Ciencia y Tecnología de 1990}

Desde el 2007, se inició una amplia discusión para debatir sobre posibles modificaciones a la Ley de Ciencia y Tecnología de 1990. Esto implicó promover un cambio integral, que planteaba temas de poder de los actores participantes. Esta transformación conllevaba cambios en juntas directivas, planificación y uso de recursos; en un contexto en el que existen diversos núcleos de poder sobre el tema.

La reforma buscó ampliar los objetivos, estimular un Sistema Nacional de Ciencia, Tecnología e Innovación, incorporar nuevos actores y regular los miembros del Consejo. Para Venegas Renauld, el asunto "constituye uno de los temas esenciales para una Comisión de Ciencia, Tecnología y Educación; el país requiere, efectivamente, de una ley para el impulso a la ciencia, la tecnología y la innovación" (Asamblea Legislativa, 2011, p. 19). Se reconoció, que la iniciativa se propuso desde años anteriores, pero que en una década no logró generar acuerdos importantes entre los ministros y exministros.

El proyecto inició el 22 de agosto de 2007 y el 27 de setiembre de 2012 estaba en proceso de llegar a plenaria, pero no se concretó. De modo que, fue archivado el 22 de agosto del 2012. No obstante, el proyecto presentaba ingenuidades, que quizás eran parte del interés de algunos actores por mantener inactiva dicha ley. Las reuniones de la Comisión transcurrieron una tras otra, como una mala cometida donde interesa la CTE, pero donde no hay acuerdos entre los actores para cambiar estructuras o ampliar financiamiento, por lo que muchos parecían no estar convencidos de la concepción de innovación impulsada.

Por lo tanto, la Ley era similar a "un vestido a la medida", pues, para unos constituye un recurso que acota las discusiones y para otros es un actor limitante para pasar de una visión lineal a una dinámica, que impacte la educación, el empleo y el desarrollo. De alguna forma, la existencia de la ley frena la articulación de nuevos actores, desvincula otros con conocimientos y experiencia en la gestión de procesos de investigación y uso de tecnologías, limita el uso de recursos y la mayoría del tiempo genera la idea de que la Comisión de Ciencia y Tecnología no es el mecanismo que puede estimular dinámicas sociales para el uso de la CTE y así mejorar la calidad de vida, generar cohesión social y sostenibilidad.

\section{Incrementar el presupuesto a la educación pública y la innovación}

En Costa Rica, cada 10 años se realiza una revisión concerniente a la educación. En la práctica, esto ha significado incluir políticas incrementales en presupuestos para esa actividad. En el 2011, la AL aprobó una reforma constitucional para invertir un 8\% del Producto Interno Bruto (PIB) en educación, lo cual significó un cambio en el artículo constitucional número 78, pasando el piso presupuestario del 6 al 8\%. Esta fue una estrategia para continuar con las prácticas de algunos países desarrollados; lo cual representa un desafío en la negociación anual entre las autoridades de los ministerios ligados al fisco, la educación y los rectores de las universidades públicas.

Aún cuando el incremento se volvió obligatorio a partir del 2014, el Ministerio de Hacienda $(\mathrm{MH})$ ha aumentado la asignación presupuestaria en forma gradual año tras año. Por ejemplo, en el 2012 se destinó al sector educativo el 7.02\%, para el 2013 el 7.2\% y el 7.42\% en el 2014. Es importante explicar, que del presupuesto destinado al sector educativo, el 1.5\% del PIB le corresponde al Fondo Especial de Educación Superior (FEES). No obstante, a futuro se requiere de una discusión sobre las proporcionalidades presupuestarias entre la educación preescolar, primaria, secundaria, técnica y la educación universitaria. 
No obstante, dentro del marco del FEES, surge un desacuerdo entre el uso de los presupuestos en los campus principales de las universidades y el destinado a apoyar las sedes y centros académicos de las regiones. Cada vez resulta más difícil justificar por qué no se invierte en investigación dirigida desde las sedes y centros hacia las comunidades y las regiones, principalmente, las que poseen mayores características rurales. Es importante plantearse cómo las rectorías de turno logran mantener agendas y presupuestos centralizados, sin lograr impactar los problemas regionales desde sus propias visiones.

Además del financiamiento englobado dentro del presupuesto nacional, se han negociado otros adicionales en el marco de los préstamos externos. Al mismo tiempo, se aprueban otros nuevos con los objetivos de "mejorar el acceso a la calidad, para incrementar las inversiones en la innovación y el desarrollo científico y tecnológico, así como actualizar la administración institucional, todo dirigido al sistema de educación superior pública" (Préstamo número 8194-CR). A través del MEP, se establece mejorar la coordinación con el CONARE, las Universidades Públicas y el Sistema Nacional de Acreditación de la Educación Superior (SINAES). Los actores estratégicos de este proceso, se aprecian en el Grafo 3.

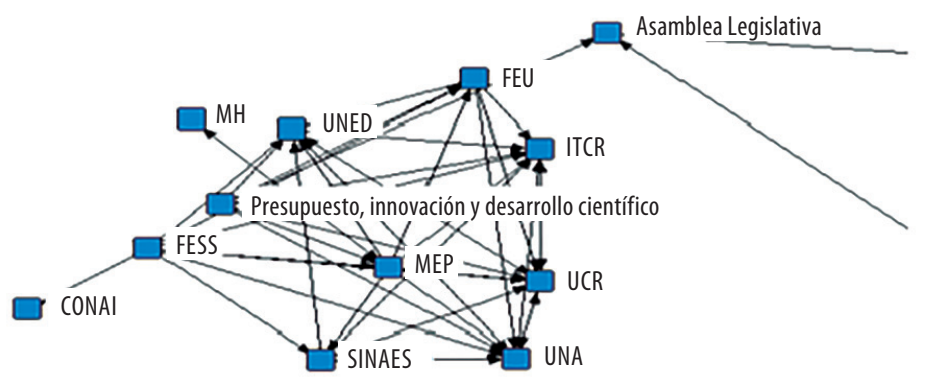

El presupuesto a la educación se ha tendido a consolidar, con el fin de que la formación del factor humano sea uno de los recursos de competitividad y cultura que puede fortalecer, hacia el futuro, el modelo democrático del país. La red muestra una gran cohesión, y cambiar la tendencia significaría volver atrás a nivel constitucional que podría darse sólo ante la amenaza de crisis fiscal o la presencia de liberales en el Congreso. No obstante, la cohesión de la red asegura la sostenibilidad del régimen logrado.

Como se puede observar, el Estado, las universidades y pocos actores en el Congreso deciden una agenda que fortalece la CTE. Sin embargo, existen interrogantes en torno a dónde se discute, dónde se invierte y dónde impacta la ciencia y la tecnología. Esto refuerza la concentración de recursos en espacios urbanos, lo cual lleva a mantener la tendencia en las migraciones de la población rural y a una caída en el dinamismo del PIB del mismo origen. Estas experiencias demandan mayor espacio a la descentralización y democratización, con el fin de que no mermen las relaciones de gestión al interior de las instituciones.

\section{Un encuentro entre tres redes}

Las redes ligadas a la Comisión Permanente de Ciencia, Tecnología y Educación, dejan ver la siguiente perspectiva cognitiva en su primer Acta:

Reflexionar, analizar, discutir y proponer legislación que contribuya a fortalecer la educación, la ciencia y la tecnología, como puntas de lanza del desarrollo social... en procura de mejorar el marco legal y con ello las oportunidades, la calidad de vida y el bienestar de niños, niñas, jóvenes en formación y de manera general la sociedad costarricense (Asamblea Legislativa, 2010, p. 3). 
Ante esto, cabe preguntarse ¿qué diagnóstico tiene la Comisión del problema?, ¿cómo se busca lograr el desarrollo social?, ¿cómo se puede penetrar en esos sectores que no tienen relación con la ejecución de diferentes programas educativos?

Cinco meses después de entrar en funciones, la Comisión se plantea sus propias preguntas sobre el rumbo a seguir, y para elaborar una visión inicial, se sugiere dar audiencias y consultar a diferentes autoridades. En primer lugar, se invita a diferentes miembros del Consejo de Educación Superior, a la Ministra de Ciencia y Tecnología y al Ministro de Cultura y Juventud, a que asistan a las sesiones. El objetivo es:

Escuchar a los diferentes actores con poder de decisión en el ámbito de la ciencia, la tecnología en Costa Rica. Se proponen a partir de las experiencias y especialización para ilustrar e instruir sobre cuáles son, a su parecer, debilidades y amenazas que afronta el ámbito científico y tecnológico costarricense. La colaboración de las autoridades está orientada a buscar, en conjunto, posibles soluciones con reformas legales que se consideren necesarias (Asamblea Legislativa, 2010, p. 5).

Esta idea de trabajo es limitada, pues supone que las instituciones tienen un diagnóstico sobre el problema y se tenía el pensamiento de que éstas logran alcanzar, en forma unísona los resultados para superarlo. Es necesario mencionar, que tales instancias retoman estos temas desde una perspectiva marginal, ya que se concentran en temas más macros de articulación a la globalización.

De las investigaciones del MICITT se desprende que Costa Rica se encontraba en la posición diez del mundo en cuanto a la capacidad individual para usar tecnologías de información y comunicación (TIC). Según su percepción, eso se debió a las decisiones tomadas a partir de la década de los noventa, cuando se decidió invertir en informática educativa, en un programa masivo de cobertura nacional que llevó computadoras a las aulas. No obstante, según la novena Acta de la Comisión, Costa Rica ocupaba el puesto veintiséis cuando se habla de la preparación de las empresas y en el puesto cuarenta y seis sobre la preparación del Gobierno (Asamblea Legislativa, 2010).

Lo anterior, señala una condición heterogénea que requiere de muchas complementariedades. El diagnóstico siempre está relacionado a otros, pues constituye una perspectiva comparada, que no es suficiente, ya que se requiere conocer el uso que le dan las instituciones y los sujetos a la tecnología, con el fin de conocer cómo cambiar las prácticas, de ser necesario. Esto se convierte en un gran espacio de trabajo para la historia, la sociología y la antropología desde una perspectiva transdisciplinaria, entendiendo esta como las interacciones y reciprocidades entre las disciplinas al interior de un sistema, permitiendo el cruce de informaciones y aboliendo las fronteras disciplinarias (Luengo González, 2012).

No obstante, los discursos proporcionan premisas para pensar que el camino es imitar esfuerzos que hacen otros países por introducir el Internet, como un símbolo de innovación y ventaja comparativa, que puede impactar las estadísticas del PIB. No obstante, en la Comisión no se discute el rostro de la pobreza, ni la forma en que las familias logran llevar sus hijos a las escuelas, ni tampoco cómo el uso de algunas tecnologías obedecen más a una dinámica de mercado que a una necesidad familiar para superar diversos problemas no resueltos.

Las reformas en el MEP iniciaron en el 2006 planteando un marco de ética, estética y ciudadanía como ejes centrales de la educación. Pero sin revisar antecedentes, la Comisión se interesa por profundizar en lo que ha venido haciendo el MEP, sin abrir el tema a otras entidades, universidades y a otros centros que ya presentaban evaluaciones sobre los avances educativos en el país.

En ese marco, la Comisión atiende las principales causas de la deserción escolar. Según las viceministras de Educación, Silvia Víquez Ramírez y Dyalah Calderón de la O, dicha deserción se pueden deber a tres razones: a) la expulsión de los estudiantes asociados al rendimiento académico, b) la pérdida de interés por falta de pertenencia, arraigo e identidad de los estudiantes y c) la exclusión debido a condiciones socioeconómicas. Para cada una de estas causas el Ministerio realizó algunas gestiones, pero en el inventario de acciones parece ser un superministerio (presenta pocas acciones de coordinación), 
excepto por los casos de $\mathrm{FONABE}^{6}$ y AVANCEMOS $^{7}$, donde se revela debilidad en la coordinación de políticas públicas con otras entidades. En el desarrollo de estrategias de articulación a nivel local para mejorar resultados. Las lógicas de coordinación y articulación son limitadas.

Existe una ligera relación con políticas sociales, pero no parece ser el fuerte del proceso. FONABE y AVANCEMOS destacan como programas fundamentales para superar las necesidades económicas; donde claramente se identifican subsistemas con oportunidades y limitaciones. En ambas, fallan elementos para conocer las asimetrías, el desempleo, la marginación y los problemas de salud y de nutrición.

La Comisión, sin profundizar en un diagnóstico, intenta estar al día y promover la idea de introducir nuevas tecnologías informáticas en todo el país. Para ello, se da atención a la carta de entendimiento entre el Gobierno de la India y Costa Rica, con el propósito de obtener recursos para construir y gestionar el Centro de Excelencia de Tecnología de la Información (CETI) en Costa Rica.

Otro tema que cobra fuerza a partir de este diagnóstico, es la construcción de infraestructura. Para esto, la Comisión se concentra en analizar la figura del fideicomiso, como una opción para dotar de instalaciones a escuelas y colegios según data en su Acta número 58:

El proyecto fue presentado (...) el 23 de setiembre (2010), la intención que tiene el Ministerio es contar con un mecanismo alternativo apropiado para acelerar la inversión e infraestructura educativa. Esto debido al déficit que (...) se cuantifica a través de encuestas realizadas, anualmente, a los directores de centros educativos, en las cuáles ellos indican las necesidades que tienen en cuanto a planta física... Este déficit tiene su origen en la obsolescencia de los edificios, la mayoría de los edificios tienen, por lo menos, arriba de veinte, treinta años de haberse construido; al incremento de la demanda, principalmente a nivel de secundaria; la diversificación de la oferta educativa y a los fenómenos naturales. Estos son los orígenes de este gran déficit que tenemos en cuanto a planta física (Asamblea Legislativa, 2012 p. 5-6).

En general las consultas de la Comisión dejan entrever que cada entidad trabaja por separado, al centralizar las decisiones sin mayor coordinación y articulación con otras entidades. Ante este grupo separado de iniciativas, aun cuando guardan relación con CTE, se presenta una amplia diversidad de temas, sin priorizar una propuesta que avance en alguna dirección. No hay prioridades claras en los discursos, sino que se suman temas sin un norte definido.

\section{¿Hacia dónde avanzan el MICITT y su red?}

Para la diputada Fonseca en el marco legal del MICITT:

(...) en el fondo el tema es la creación de un ministerio que viene a facilitar, a promover a la investigación científica y la innovación tecnológica, todo lo que tiene que ver con la transferencia al sector productivo (...) Luego (...) otro elemento es incorporar la ciencia y la tecnología y la innovación a los procesos productivos y luego laborar en un marco de solidaridad, eficacia y transparencia. Hemos hecho un esfuerzo muy importante en esta línea y promover, en general, el desarrollo y la calidad de vida de los costarricenses (Asamblea Legislativa, 2010, p. 4).

6 FONABE (Fondo Nacional de Becas) facilita becas para dar continuidad al estudio.

7 Es un Programa Social de Gobierno donde intervienen varias instituciones, ejecutado por el Instituto Mixto de Ayuda Social (IMAS). Su objetivo es promover la permanencia y reinserción en el sistema educativo formal de adolescentes y jóvenes pertenecientes a familias que tienen dificultades para mantener a sus hijos en el sistema educativo debido a problemas económicos. 
Grafo 4

Actores en la agenda de ciencia, tecnología y educación en el año 2010

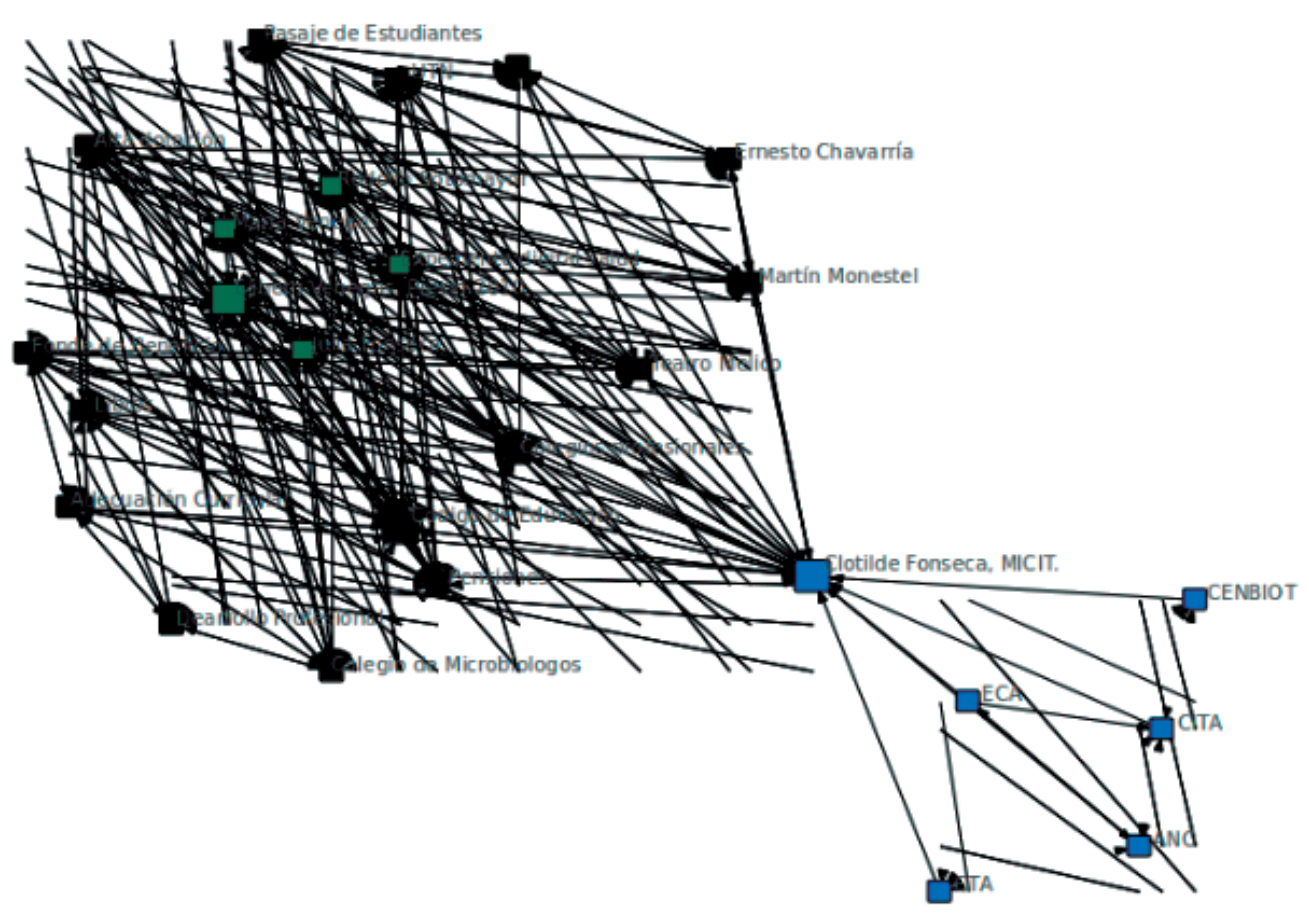

El MICITT coordina una agenda con otros actores que se aprecian en el Grafo 4. Se reconoce en el acta legislativa que los presupuestos disponibles son "impresionantemente bajos (...) la preocupación es ¿cómo podemos fortalecer esta área del desarrollo? Porque Costa Rica, como país, no va a poder llegar realmente a un desarrollo pleno y valioso sino tenemos una inversión mayor" (Asamblea Legislativa, 2010, p. 6).

Efectivamente, el presupuesto resulta simbólico para hacer frente a los diferentes desafíos asumidos por el MICITT. La Ministra Clotilde Fonseca Quesada reconoce que:

\footnotetext{
La investigación en ciencia y tecnología en el país, (...) es la inversión en enseñanza y formación, luego en investigación y desarrollo y luego en servicios científicos. En la distribución de la investigación y desarrollo hay un pequeño sector de la sociedad civil que invierte, hay un sector más grande, que es el sector público, que ahí hay inversión importante, especialmente, en ministerios más amplios o en instituciones como la Caja del Seguro Social (CCSS), el Ministerio de Agricultura (MAG), el Instituto Costarricense de Electricidad (ICE), y se reconoce que las empresas tienen un poco de inversión. Se considera que en Costa Rica todavía es muy limitada la inversión de las empresas; y la inversión del sector académico es mucho más grande (Asamblea Legislativa, 2010, p. 8).
}

Pese a esto, resulta difícil el encuentro con otros actores que, como usuarios de las TIC, requieren estandarización y apoyo para el uso de sistemas más adecuados; lo cual constituye un tema transversal para instituciones y empresas. Curiosamente, se plantea que las empresas invierten poco en investigación y que sólo las universidades lo hacen, aseveración que invita a estimular nuevas reflexiones. Así, los gerentes de entidades públicas y empresas privadas trabajan en equipos que lideran procesos de producción de bienes y servicios, pero que se apoyan en el uso de las TIC para mejorarlos. En diferentes ámbitos de las zonas rurales y las urbanas, parece que la industria utiliza intensamente estas tecnologías. 


\title{
Las redes de las universidades públicas
}

Estas redes coordinan esfuerzos especialmente en la lucha presupuestaria frente al PE intermediado por el FEES, donde las negociaciones las organiza el MEP y el MH. En el 2012 se acordó, adicionalmente, obtener un préstamo de 200 millones de dólares con el fin de responder al rezago histórico de la inversión en infraestructura, equipamiento y formación de recursos humanos y el desarrollo de la ciencia y la tecnología en áreas prioritarias.

En el Expediente Legislativo $\mathrm{N}^{\circ} 18700$, se plantea que:

\begin{abstract}
El desarrollo de la educación superior en los últimos años ha estado caracterizada por el hecho de que las universidades estatales, como resultado de su gestión, han establecido gradualmente determinadas relaciones con la sociedad que implican asumir compromisos mutuos con el propósito de contribuir, de un modo eficaz, al cumplimiento de su misión social" (Asamblea Legislativa, 2013b, p. 1).
\end{abstract}

De este modo, se establece que la universidad tiene relaciones con su entorno, creadas a partir de demandas sociales en relación con los resultados de sus procesos. Aparecen las universidades públicas como entidades pertinentes, debido a que se articulan programas y proyectos tanto en el Gobierno, como en otras organizaciones.

A partir de esto, se puede decir que:

\begin{abstract}
El concepto de pertinencia conlleva también la capacidad de las universidades de asumir críticamente los retos que demanda la sociedad en su conjunto, para contribuir con ello a su transformación. Ello supone, no solo la pertinencia económica, sino también la pertinencia social y cultural, así como el papel activo de las universidades como agente de cambio de nuevas ideas y acciones dirigidas a lograr una sociedad mejor (Asamblea Legislativa, 2013b, p. 1).
\end{abstract}

En esta misma perspectiva se da la idea de que las universidades logran avanzar en la innovación, la investigación y la creatividad, con lo cual se facilitan procesos de erradicación de la pobreza, el desarrollo sostenible y se puede avanzar hacia los Objetivos del Milenio (ODM).

No obstante, se sigue reproduciendo la idea de que la innovación es un proceso lineal que se alcanza a partir del vínculo entre universidades y sociedad. Sin embargo, esta situación presenta un alto grado de incertidumbre, pues demanda muchos recursos, genera invenciones y muchos aportes. A pesar de esto, no siempre se logra la innovación y, en pocas experiencias, se impacta la comunidad o la empresa con nuevos desarrollos o potencialidades. En muchos casos, los esfuerzos presentan un tiempo y espacio limitados, por lo que se deja o se abandona la escena con más rapidez de lo esperado.

En el fondo, más que influir directamente en indicadores sociales, los mayores avances están en el fortalecimiento institucional de la universidad. Ejemplo de ello se da al incrementar la matrícula, mejorar la calidad de los programas y la infraestructura y aumentar el número de carreras acreditadas.

El desafío de generar aportes y relaciones entre universidades y empresas para aumentar la innovación constituye un tema complejo, pues formular políticas de investigación, de extensión y de acción social es materia de discusión interna y se realiza de forma cerrada a la sociedad. Las universidades no construyen sus agendas en una forma abierta con los sectores productivos y las comunidades, pues son limitadas las experiencias exitosas de este tipo. Por lo tanto, debe realizarse un nuevo acercamiento. Desde el sector académico, es necesario promover la vinculación con los sectores productivos, las comunidades y el gobierno. La agenda universitaria, no sólo debe ser construida tomando como base los criterios de estos actores, sino que, debe promover también el diseño de una agenda en conjunto con el propósito de motivar no sólo la generación del conocimiento, sino también la satisfacción y la resolución de las necesidades de cada uno de los sectores sociales.

\section{Redes para la competitividad e innovación desde el PE}

El PE también centraliza una agenda ligada a la tecnología y la innovación. Estas redes articulan diferentes actores institucionales y a un particular sector productivo. En su diagnóstico tiene presente 
que el crecimiento del país obedece a un aumento en el uso de los factores de producción, manteniendo la productividad estancada. Además, los salarios y las cargas sociales son altos para poder competir en actividades intensivas en mano de obra de baja calificación. Ante esto, se sugiere seguir estrategias foráneas y analizar la participación en mercados internacionales.

Para esta red, el país no tiene las mejores condiciones para desarrollar su capacidad de innovación. Los elementos de la competitividad se logran a partir de las instituciones, las redes políticas y otros factores, los cuales determinan el nivel de productividad y el crecimiento a mediano y largo plazo. El argumento es que sólo con mayores productividades se pueden mejorar las fuentes de empleo y los salarios de los trabajadores. Sin embargo, aumentar el ingreso que percibe cada uno de los trabajadores puede poner en riesgo niveles de competitividad de las empresas, es decir, éstas no pueden ahorrar ni invertir.

Los problemas para la competitividad e innovación en Costa Rica, de acuerdo con los diversos diagnósticos, son: insuficiente desarrollo de capital humano e innovación, la necesidad de desarrollar más el comercio y atraer más inversión externa, más financiamiento y desarrollo de un mercado de capitales, la falta de desarrollo de infraestructura y la urgencia de simplificar trámites.

Esta visión central se impone a las otras y hace compleja la posibilidad de generar nuevas investigaciones para determinar cuáles son los problemas por sector y por regiones, y así determinar la forma en que deben articularse los capitales sociales.

\section{Conclusiones}

Esta investigación muestra el encuentro entre diversas redes, que plantean inquietudes importantes sobre el futuro próximo de la CTE, así como la formación de factor humano, la migración rural-urbana, el empleo, el ejercicio de la coordinación de agendas entre redes e instituciones públicas y privadas, la competitividad y el desarrollo.

Las reformas de la Ley de Ciencia y Tecnología en Costa Rica serán cada vez más difíciles, pues la estructura favorece a los partidos políticos en el poder. Además, ésta es una ley sumamente estratégica, en cuanto a dirigir recursos para estimular nuevos ejes de tecnología e innovación, pero con presupuestos limitados ante la imposibilidad de una reforma fiscal. Sólo el surgimiento de desafíos en la gobernanza de las instituciones relacionadas con la red dará espacio para la modificación. En materia de CTE, las agendas deben ser pensadas desde la estrategia país a largo plazo y no desde las administraciones del gobierno de turno; no obstante, la legislación no facilita esta perspectiva.

Al coexistir varias agendas se complica la eficacia y eficiencia, lo que afecta la gobernanza institucional; por lo tanto, coordinar se hace difícil de realizar. La descoordinación sigue manteniendo un poder central en la gestión, y una ineficacia en la asignación de fondos y acciones para que las regiones mejoren su dinamismo. La innovación hoy más que nunca es social, depende de múltiples actores, es relacional y, en gran medida, está cerca de aquellos que se enfrentan día tras día con procesos para mejorar servicios y productos.

La red de educación muestra una cohesión importante, que lleva a pensar que el régimen alcanzado tiende a una sostenibilidad que sólo puede ser afectada por visiones liberales que busquen reducir el gasto del Gobierno y el déficit. Esto ocurre sólo ante crisis fiscales calificadas, o cambios entre los partidos que tradicionalmente llegan al poder.

Los diagnósticos que relacionan la educación, la pobreza, el acceso a la tecnología y la coordinación interinstitucional a nivel central y regional son limitados, por lo tanto, la Comisión Legislativa y los ministerios parten de sesgos importantes al diseñar iniciativas para mejorar la educación. En este sentido, existe descoordinación a nivel nacional y regional y no hay una visión articulada. Esto dificulta el uso de la ciencia, la tecnología y la innovación para generar cambios sociales orientados hacia la cohesión social, la sostenibilidad y la gobernanza; especialmente en regiones rurales. 
El MICITT, como ente rector, mantiene su acción limitada a una red que no representa los problemas que enfrenta la sociedad, en una nueva etapa de uso de la tecnología y la información como base indispensable para la toma de decisiones. Tanto las instituciones como las empresas privadas hacen uso de diferentes herramientas tecnológicas para mejorar su conocimiento, tomar decisiones y mejorar los procesos productivos.

Las experiencias de las universidades deben ser fortalecidas para que puedan abrir sus agendas a la sociedad y articular acciones que respondan a las distintas necesidades. Es fundamental encontrar mejores caminos de coordinación institucional entre universidades y las empresas privadas y entre ambas con las regiones productivas del país; con el fin de generar mayor impacto.

El PE parece ser el único actor capaz de abrir una agenda y llegar a acuerdos básicos que permitan mejorar el uso de recursos y la articulación entre actores estratégicos que al final son los gestores directos del cambio. Sin embargo, esto depende del liderazgo y de las agendas del PE y de su relación con la AL y la Comisión. No obstante, esto no se percibió en este estudio; el espacio político es limitado, pero parece ser ésta la estrategia del mismo Congreso, para mantener la posibilidad de hacer política pública sin hacerla.

Desde los aportes teóricos dados por Gomá y Subirats (1998) se puede afirmar que en el caso de Costa Rica, las principales redes de CTE presentan propuestas muy cerradas dentro de la esfera formada por los poderes Legislativo y Ejecutivo. Sólo en el caso del PE hay una cercanía con el sector privado; por lo cual se presenta una limitada coordinación entre la esfera pública y la privada.

\section{Agradecimientos}

Los autores de este trabajo agradecen a la Vicerrectoría de Investigación de la Universidad de Costa Rica (UCR), por apoyar las actividades desarrolladas dentro del marco del proyecto VI805-B3218: "Construcción histórica de las políticas públicas de la ciencia y tecnología en Costa Rica 1972-2010- Ciencia cohesión social en Costa Rica”, perteneciente al Programa de Estudios Sociales de la Ciencia, la Técnica y el Medio Ambiente (PESCTMA) (VI-805-A4906) del Centro de Investigaciones Geofísicas (CIGEFI) de la UCR.

\section{Referencias}

Aguilar, Luis F. (1996). La hechura de las políticas. México: Miguel Ángel Porrúa.

Arellano, Antonio. (2003). La sociología de las ciencias y de las técnicas de Bruno Latour y Michel Callón. Cuadernos Digitales: publicación electrónica en historia, archivística y estudios sociales, 8(23).

Barrantes, Rodrigo (2001). Investigación: Un camino al conocimiento, un enfoque cualitativo y cuantitativo. San José: EUNED.

Doménech, Miquel y Tirado, Francisco. (2008). El problema de la materialidad en los estudios de la ciencia y la tecnología. España: Universidad Autónoma de Barcelona.

Grau, Marc., Iñiguez-Rueda, Lupicinio, \& Subirats, Joan. (2010). "La perspectiva sociotécnica en el análisis de políticas públicas". Rev Psicología Política, (41), 61-80.

Gomá, Ricard. y Subirats, Joan. (1998). Políticas públicas en España. Contenidos, redes de actores y niveles de gobierno. Barcelona: Ariel.

Latour, Bruno. (1991). La Tecnología es la Sociedad Hecha Para que Dure. En M. Domènech y F. Tirado (eds.) Sociología Simétrica. Ensayos sobre ciencia, Tecnología y Sociedad (pp. 109-142). Barcelona, Gedisa.

Latour, Bruno. (2005). Reassembling the social: an introduction to actor-network theory. Nueva York: Oxford University Press. 
Law, John. (1998). "Del poder y sus tácticas. Un enfoque des de la sociología de la ciencia”, en M. Domènech y F. Tirado (eds.). Sociología Simétrica. Ensayos sobre ciencia, Tecnología y Sociedad (63-107). Barcelona: Gedisa.

Luengo González, Enrique. (2012). La transdisciplina y sus desafíos a la universidad. En Interdisciplina y transdisciplina: aportes desde la investigación y la intervención social universitaria (pp.9-25). Guadalajara, México: ITESO.

Ministerio de Ciencia y Tecnología (MICIT). (2011). Plan Nacional de Ciencia, Tecnología e Innovación 2011-2014. Ministerio de Ciencia y Tecnología (MICIT).

\section{Documentos del congreso consultados:}

Asamblea Legislativa. (2010-2014). Comisión Permanente de Ciencia, Tecnología y Educación. Actas 1 a la 111.

Asamblea Legislativa. (2014). Reglamento de la Asamblea Legislativa de Costa Rica: con resoluciones de la Sala Constitucional de la Corte Suprema de Justicia, resoluciones de la Presidencia de la Asamblea Legislativa y criterios del Departamento de Servicios Técnicos (Segunda Edición). San José, Costa Rica: Departamento de Servicios Bibliotecarios, Documentación e Información. Asamblea Legislativa. (2013a). Aprobación del contrato de préstamo No. 2852/OC-CR suscrito entre la República de Costa Rica y el Banco Interamericano de Desarrollo para financiar el programa de innovación y capital humano para la competitividad. Expediente 18773.

Asamblea Legislativa. (2013b). Aprobación del contrato de préstamo № 8194-CR suscrito entre la República de Costa Rica y el Banco Internacional de Reconstrucción y Fomento para financiar el Proyecto de Mejoramiento de la Educación Superior. Expediente 18700. 
